\title{
EL USO DE RECURSOS MULTIMEDIA EN LA REPRESENTACIÓN DEL TERRITORIO: EL "ATLAS MULTIMEDIA DE GEOGRAFÍA DE ARAGÓN"
}

\author{
Severino ESCOLANO UTRILLA y Enrique RUIZ BUDRÍA \\ Departamento de Geografía y Ordenación del Territorio \\ Universidad de Zaragoza
}

\begin{abstract}
Resumen: El "Atlas multimedia de geografía de Aragón" es una aplicación desarrollada en el Departamento de Geografía de la Universidad de Zaragoza, con finalidad docente y para divulgar información geográfica a escala regional. En este artículo se describen y valoran el sistema de navegación y las herramientas diseñadas para aprovechar las capacidades de almacenamiento y procesado de información de la tecnología informática.
\end{abstract}

Palabras clave: Multimedia, información territorial, atlas electrónico, escala regional.

\begin{abstract}
The "Multimedia Atlas of Aragon" is an application developped in the Department of Geography at the University of Zaragoza; it's devoted to teach and to publish geographical information at regional escale. In this paper We describe and value the navigation system and the tools built in order to take advantage of the capabilities of information tecnology.
\end{abstract}

Key words: Multimedia, territorial information, electronic atlas, regional scale.

El "Atlas multimedia de geografía de Aragón" es el resultado de un proyecto de investigación ${ }^{1}$ desarrollado en el Departamento de Geografía con la colaboración del Servicio Multimedia de la Universidad (SMUZ)2. Es, en esencia, una aplicación que combina algunas capacidades gráficas y de procesado de información de los ordenadores, para visualizar y manejar datos territoriales en un entorno multimedia.

1 El "Atlas multimedia de geografía de Aragón" es fruto de un proyecto de investigación financiado por la Caja de Ahorros de la Inmaculada y la Institución "Fernando El Católico" de la Diputación de Zaragoza.

2 Además de los autores de los mapas, han intervenido los geógrafos M. Espallargas y M. M. Martínez, responsables de la edición de los mapas; la programación se ha efectuado en el SMUZ y es obra de I. Ferrando en colaboración con E. Casasín 
A pesar de que los atlas electrónicos proliferan en Europa Occidental y Estados Unidos, en España todavía no han iniciado el despegue; aunque no es lugar para exponer las razones de este retraso, si que cabe mencionar la escasez de información geográfica digital, a escalas grande y media y a precio razonable, como una de las causas principales. Al carácter pionero de nuestro trabajo se añade otra nota distintiva, pues es uno de los pocos realizados fuera del ámbito de la empresa privada y el único, que sepamos, llevado a cabo en el seno de un departamento universitario de Geografía.

Aunque no es el primer trabajo de esta índole emprendido en el Departamento de Geografía, sin duda éste es el de mayor envergadura, por la extensión de los contenidos, por el volumen de la programación y por la diversidad del equipamiento informático involucrado. Para llevar a buen puerto este proyecto ha sido necesario idear, a veces sobre la marcha, esquemas de trabajo ad hoc para bregar con la complejidad de tareas; la adquisición de un bagage -experiencia- en relación con lo dicho es un aspecto positivo no menor, de elevado valor práctico para otras empresas similares.

\section{OBJETIVO: DIFUNDIR INFORMACIÓN GEOGRÁFICA REGIONAL EN NUEVO SOPORTE Y ESTILO}

El principal objetivo del proyecto es proporcionar a los estudiantes universitarios y de enseñanza media, y al público en general, un corpus de información territorial debidamente estructurada y presentada, que sea útil para la adquisición de conocimiento geográfico. Asimismo, es un instrumento de interés para la planificación territorial, cuando se aborden asuntos que precisen datos a escala municipal. El proyecto ha producido también alguna externalidad de importancia, como es la formación de una base digital de información georreferenciada, provechosa en la docencia y posteriores investigaciones (Fig. 1).

Esta variedad de usuarios y objetivos, las escalas empleadas, la naturaleza de la materia a tratar, así como los medios implicados, han orientado la selección de contenidos y han inspirado los principios pedagógicos y metodológicos que guían este proyecto. Entre ellos destacamos:

-el enfoque temático dominante; 
Figura 1.- Atlas de geografía de Aragón: objetivos

Objetivos:

- Representar los elementos básicos del territorio aragonés

- Reflejar las estructuras territoriales

- Modelizar llo el esquema básico de organización del territorio

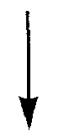

- Información para decisiones de ordenación del territorio

- Construir las bases digitales para formar un SIG regional para uso en docencia e investigación
Destinatarios:

- Estudiantes

- Técnicos en planificación territorial

- Público en general
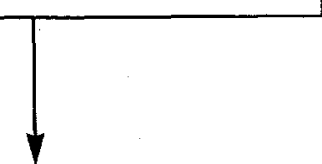

Orientaciones:

- Mapas de síntesis

- Organizaciones de contenidos sencilla e intuitiva

- Escala 1/1.250.000

- Digitalización desde mapas $1 / 300.000$

- Claridad en la cartografía

- Complejidad media alta de la información

- Edición en color

- Integrados en soporte multimedia 
-la capacidad exploratoria, que incita al usuario a examinar, de forma flexible, las distribuciones espaciales, y

-las escalas de trabajo, que permiten la cartografía de los fenómenos con cierto grado de detalle sin perder las pautas regionales de su distribución espacial.

La vía metodólogica seguida para alcanzar las metas trazadas ha consistido, básicamente, en diseñar un sistema de navegación sencillo e intuitivo, pero potente a la vez, y dotar a la aplicación de herramientas de cierta sofisticación para gestionar mapas, gráficos, textos y otras piezas de información.

\section{EL PROCESO DE PRODUCCIÓN: LARGO Y COMPLEJO}

El magnitud de la información manejada y los requisitos técnicos exigidos por los modelos y estructuras de datos, han aconsejado la división interna del trabajo para asegurar una productividad mínima.

El recorrido de los datos y el tratamiento que de ellos se hace, desde que son facilitados por los autores hasta que se convierten en mapas digitales, se esquematiza en la Figura 2. Como se aprecia, el camino es largo y en cada etapa de este tránsito se aplican las transformaciones precisas con las herramientas informáticas adecuadas, para generar los archivos que se necesitan en la siguiente estación. A su vez, cada fase comprende varias operaciones de naturaleza diversa -analíticas, cartográficas, de gestión de base de datos-, como se ejemplifica para el caso del diseño y realización cartográficos (Fig. 3).

En fin, para asegurar el uso eficiente de la información generada, se ha formado una base de datos que contiene más de mil registros de los archivos incluidos en el "Atlas".

\section{EL MAPA TEMÁtico: CARTA DE NAVEGACIÓN POR EL ESPACIO INFORMACIONAL GEOGRÁFICO}

Todos los componentes del sistema, los informativos y las herramientas, se ordenan en torno a los mapas. 


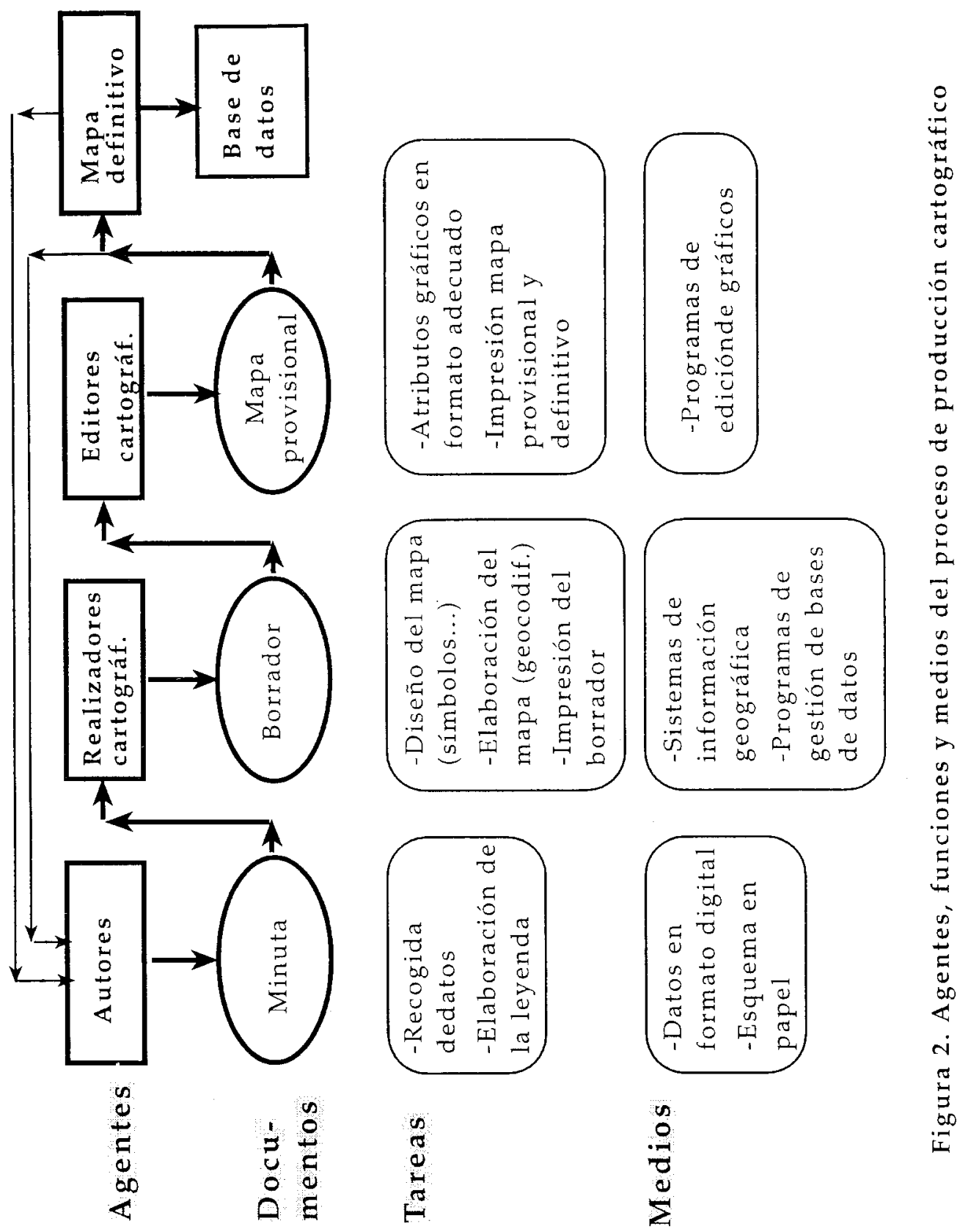


Figura 3. Etapas y funcĩones del proceso de diseño cartográfico

Fases

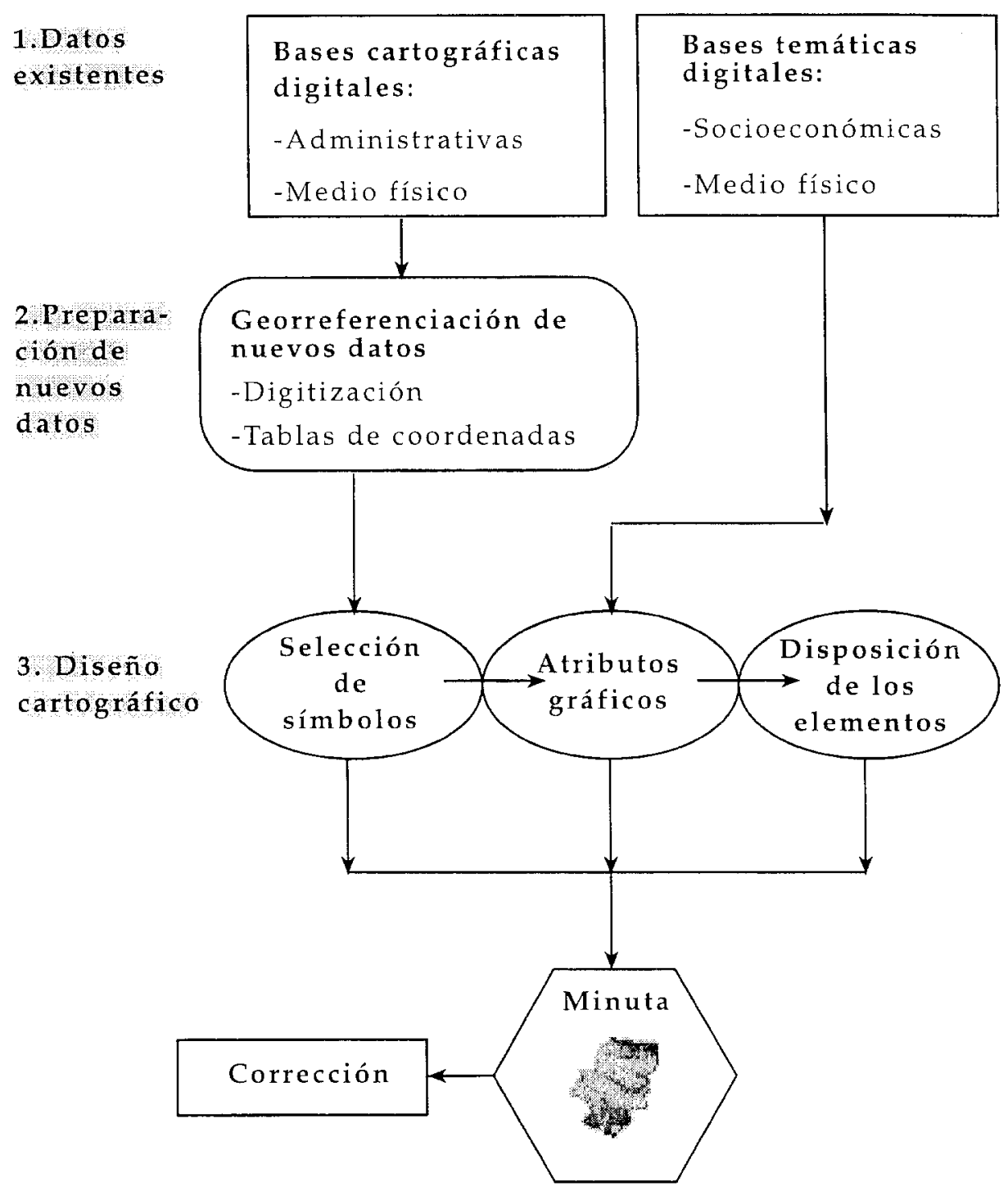


Los componentes están dispuestos de forma tal que no se exige al usuario esfuerzo para la comprensión de su funcionamiento, a fin de que pueda dedicar toda su atención a explorar los datos. A cambio, se prescinde del gran potencial analítico que caracteriza a los sistemas de información geográfica.

Los contenidos pueden calificarse de "clásicos" en lo tocante a los principales enunciados y su estructura, así como al tipo de mapas, salvo los experimentos llevados a cabo con algunas formas de simbolización cartográfica (esferas, simulación tridimensional, fusión de mapas).

El sistema de navegación diseñado al efecto otorga, en cambio, gran libertad de movimientos por el espacio informacional, pues permite no sólo relacionar los mapas con los datos y otros elementos informativos, sino también el cambio de la escala de los mapas y la ejecución de simulaciones dinámicas. $Y$ todo integrado en un entorno de trabajo sencillo e intuitivo: es ésta la aportación, original hasta cierto punto, más relevante del "atlas".

El desplazamiento sobre mapas y demás objetos a ellos subordinados, se realiza a través de menús jerárquicos, cuyos nodos finales son representaciones en miniatura de los mapas.

Una vez que el mapa seleccionado ocupa la zona de visualización, están disponibles los iconos que representan otras piezas del mapa (escala, leyenda, textos, gráficos...) o bien los que activan herramientas (Fig. 4).

Así, es posible conocer la escala y la leyenda, leer un texto, que a su vez acarrea gráficos, sobre el tema del mapa, y consultar las entradas de un glosario, los signos convencionales y los datos de una o varias tablas pertinentes al tema cartografiado en el mapa principal.

La información se completa con una selección bibliográfica, dotada de un procedimiento para búsquedas por palabras clave, establecidas en consonancia con los apartados que estructuran los contenidos. Estas partes componen un todo informativo coherente, articulado en torno al mapa temático, cuya examen creativo se facilita por las vías e instrumentos provistos para tal fin. 


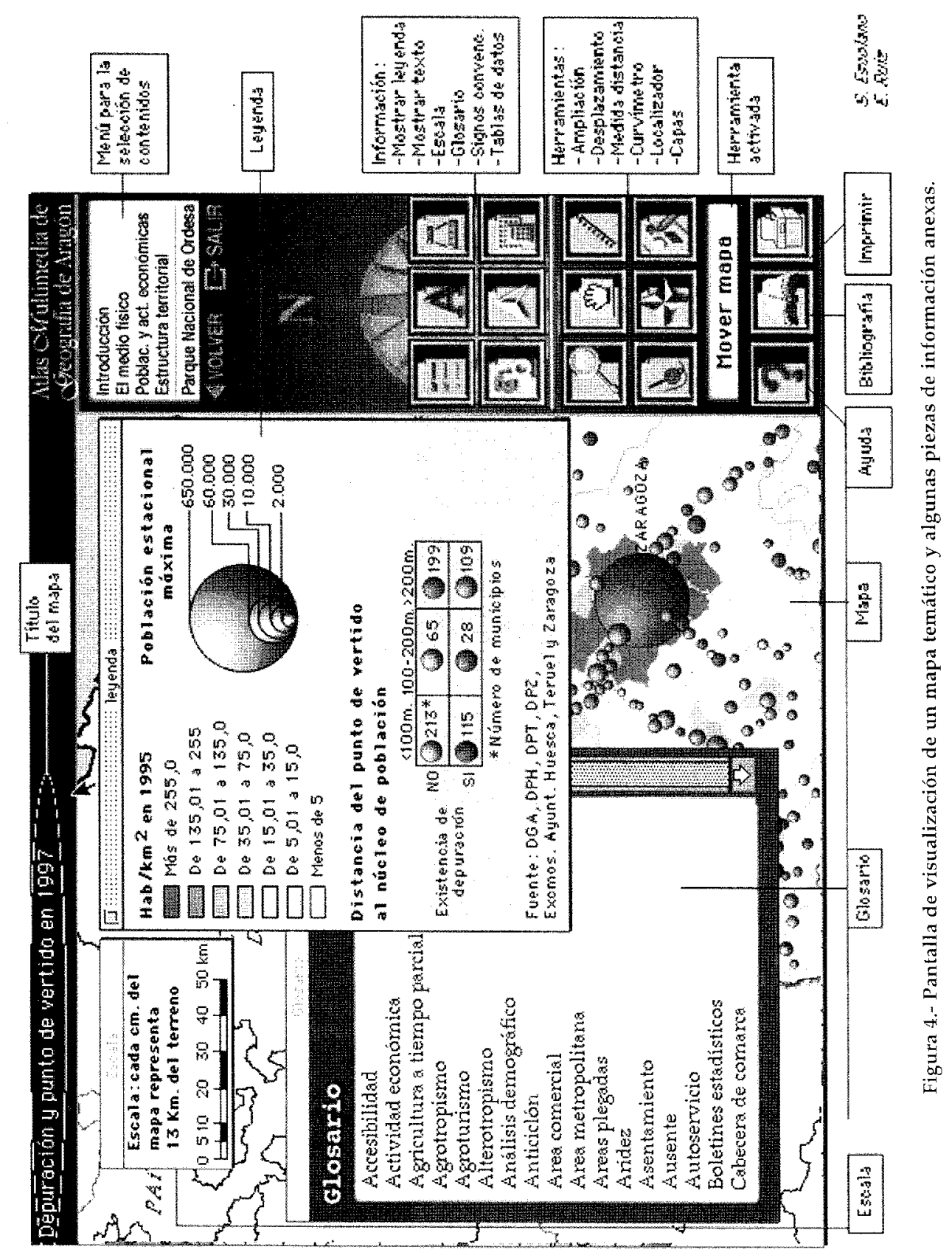




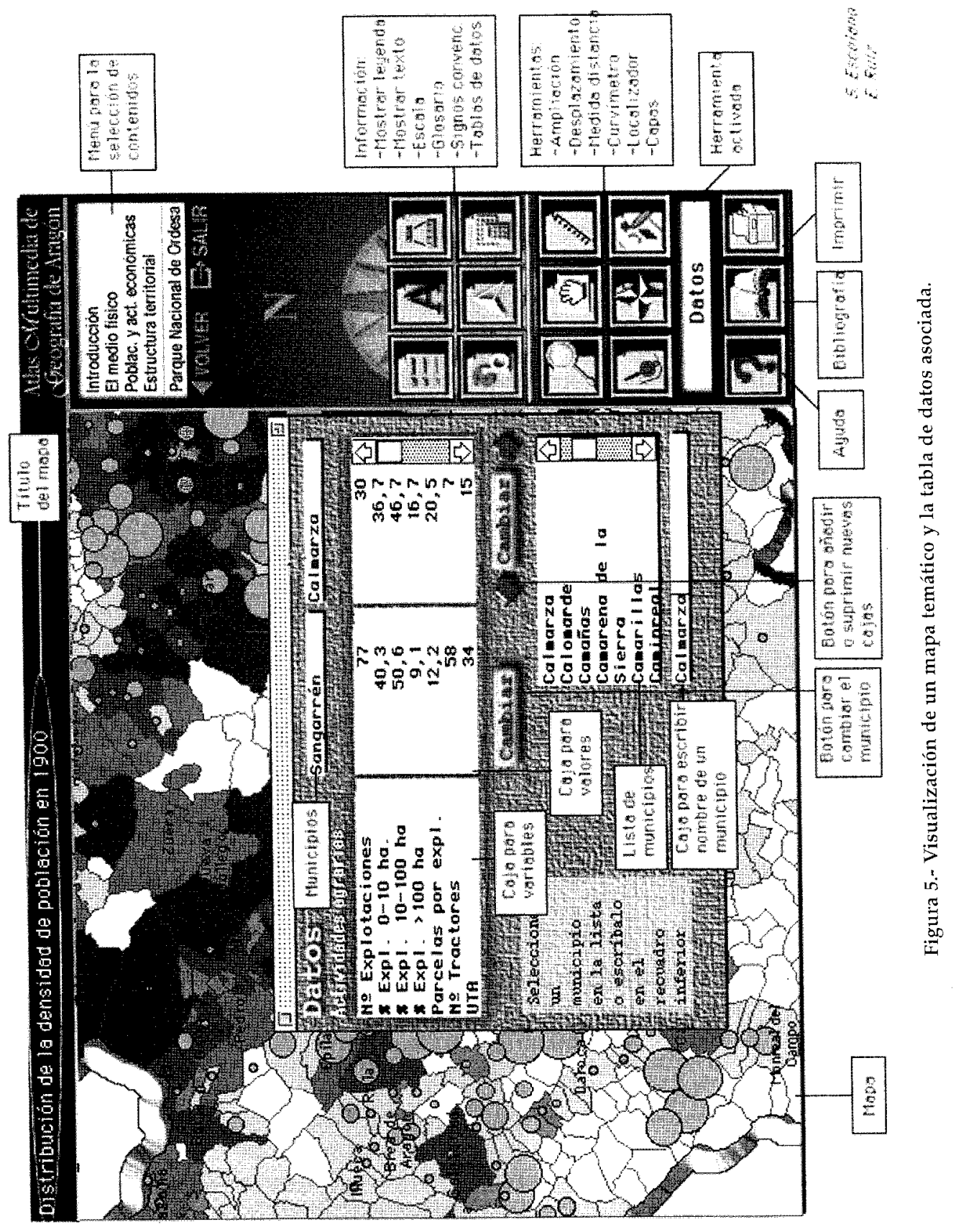




\section{HERRAMIENTAS PARA LA GESTIÓN DE DATOS: PERTRECHOS PARA LA EXPLORACIÓN}

Otra diferencia de este atlas con los atlas "convencionales", además del carácter multidimensional tratado en el parágrafo anterior, se encuentra en la disponibilidad de funciones de cierta potencia para visualizar mapas o superponer a los mismos capas de información.

La "caja de herramientas" contiene utilidades para medir la distancia, localizar los municipios, presentar los datos de una tabla, cambiar de escala y apilar estratos de información sobre los mapas. En futuras ediciones se ampliará esta batería con nuevo procedimientos de análisis espacial y de bases de datos, pero siempre concebidos para su uso sencillo e intuitivo.

Como es sabido, la distancia es una variable fundamental para la comprensión y explicación de las distribuciones espaciales. Sobre el mapa se puede medir la longitud entre dos o más puntos, en línea recta o siguiendo un trazado curvo, como el perímetro de un municipio o el cauce de un río.

El "localizador" es una rutina que rastrea la posición del cursor para determinar en que área se encuentra y mostrar su nombre, y en dirección inversa, dibuja una señal en el mapa sobre el municipio escrito en una caja de texto o marcado en una lista. Este dispositivo sirve de enlace con las tablas de datos; son éstas estructuras en forma de matriz cuyas tuplas definen los municipios mediante un número variable de campos que almacenan los valores de los atributos. La presentación de los datos se realiza en listas desplazables mediante la selección del municipio correspondiente; para fines comparativos se puede ampliar el espacio de visualización añadiendo nuevos casos (Fig. 5).

Los mapas se han construido en tres escalas: $1 / 2.100 .000,1 / 1.300 .000$ y $1 / 940.000$ aproximadamente, para presentar en la pantalla el mapa completo $y$, posteriormente, ampliar sus dimensiones a la vez que se añade nueva información.

Por fin, se ha elaborado un archivo de capas de información,- sobre el medio físico, administración y transporte- que, a modo de transparencias, se pueden apilar a discreción sobre los mapas. Es evidente la extraordinaria utilidad de esta opción para explorar asociaciones en las distribuciones espaciales (Fig. 6). 

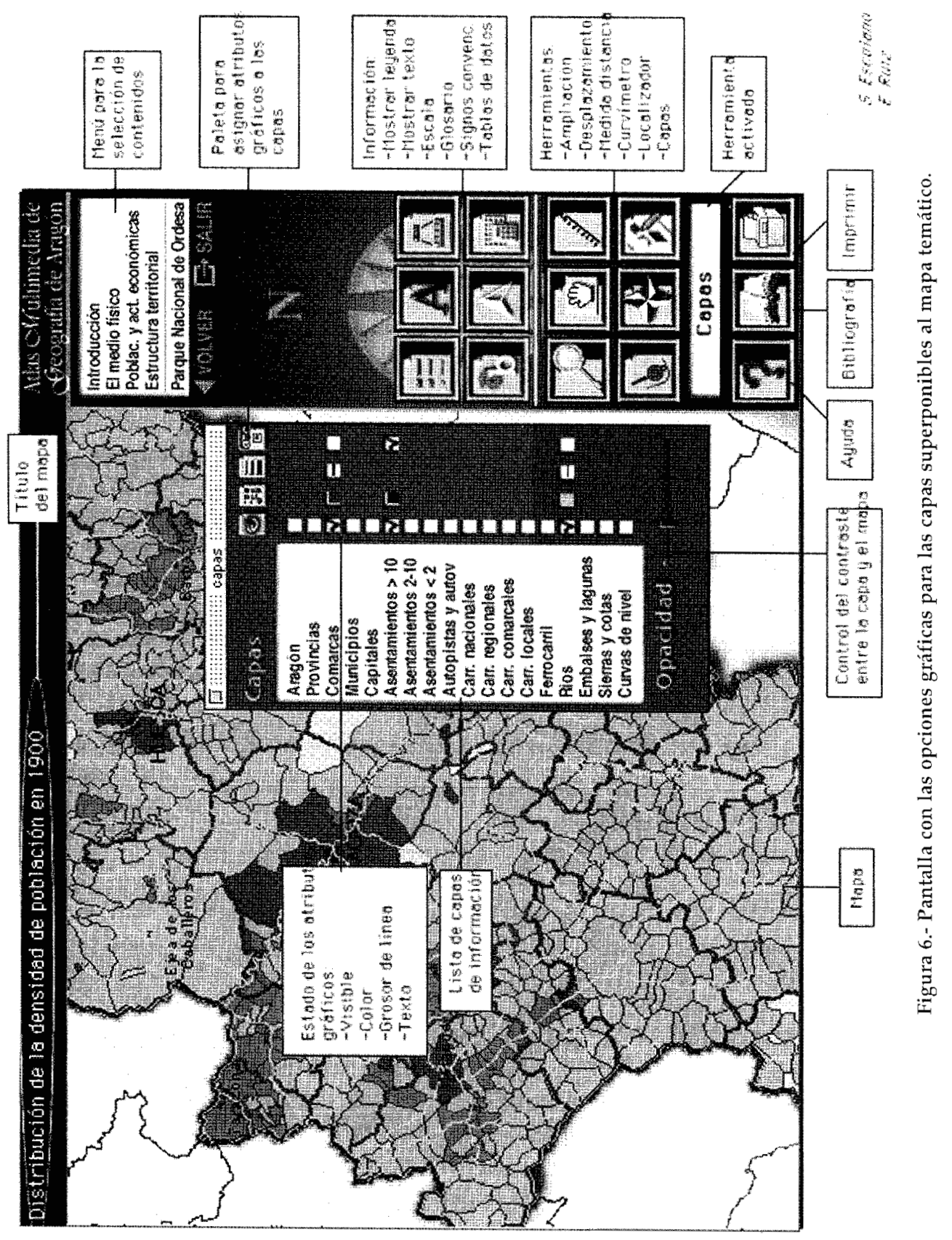
Con estas herramientas y el sistema de navegación, este atlas pretende ir más allá de donde lo hacen algunas aplicaciones comerciales, que son meras secuencias de mapas acompañados de un índice de topónimos y un procedimiento para su localización. Nosotros hemos pretendido proporcionar medios para el manejo personal de la información, pero simplificando el funcionamiento para que el usuario se concentre en los mapas, que deben ser el principal objetivo de atención.

\section{CONCLUSIONES}

La ausencia de teoría y métodos contrastados dificulta sobremanera la construcción de aplicaciones multimedia sobre aspectos territoriales. Precisamente, en esta modalidad de investigación se hace patente la relación dialéctica entre investigación teórica y praxis: es perentorio elaborar teoría sobre los nuevos estilos de visualización -simulación tridimensional, animación...- que causan impresiones perceptuales propias y diferentes de las inducidas por los modelos "clásicos", así como sobre sistemas de gestión de la información y, a la vez, es indispensable llevar a la práctica estos logros en productos concretos, que permiten afirmar o rechazar aquélla. Finalmente, y es otra cuestión relevante, podemos afirmar que estos métodos no excluyen a los tradicionales sino que ambos se refuerzan mutuamente. Sólo debemos aprender, lo que no es poco, a combinarlos en un equilibrio sabio y fecundo. 